\title{
Evading Quantum Mechanics: Engineering a Classical Subsystem within a Quantum Environment
}

\author{
Mankei Tsang ${ }^{1,2,3, *}$ and Carlton M. Caves ${ }^{3,4}$ \\ ${ }^{1}$ Department of Electrical and Computer Engineering, National University of Singapore, 4 Engineering Drive 3, Singapore 117583 \\ ${ }^{2}$ Department of Physics, National University of Singapore, 2 Science Drive 3, Singapore 117551 \\ ${ }^{3}$ Center for Quantum Information and Control, University of New Mexico, MSC07-4220, Albuquerque, New Mexico 87131-0001, USA \\ ${ }^{4}$ Centre for Engineered Quantum Systems, School of Mathematics and Physics, The University of Queensland, \\ St. Lucia, Brisbane 4072, Australia \\ (Received 26 March 2012; published 10 September 2012)
}

\begin{abstract}
Quantum mechanics is potentially advantageous for certain information-processing tasks, but its probabilistic nature and requirement of measurement backaction often limit the precision of conventional classical information-processing devices, such as sensors and atomic clocks. Here we show that, by engineering the dynamics of coupled quantum systems, it is possible to construct a subsystem that evades the measurement backaction of quantum mechanics, at all times of interest, and obeys any classical dynamics, linear or nonlinear, that we choose. We call such a system a quantum-mechanics-free subsystem (QMFS). All of the observables of a QMFS are quantum-nondemolition (QND) observables; moreover, they are dynamical QND observables, thus demolishing the widely held belief that QND observables are constants of motion. QMFSs point to a new strategy for designing classical information-processing devices in regimes where quantum noise is detrimental, unifying previous approaches that employ QND observables, backaction evasion, and quantum noise cancellation. Potential applications include gravitational-wave detection, optomechanical-force sensing, atomic magnetometry, and classical computing. Demonstrations of dynamical QMFSs include the generation of broadband squeezed light for use in interferometric gravitational-wave detection, experiments using entangled atomic-spin ensembles, and implementations of the quantum Toffoli gate.
\end{abstract}

Subject Areas: Optics, Quantum Physics, Quantum Information

According to quantum mechanics, a measurement of the position of an object must introduce uncertainty to its momentum, called the measurement backaction noise. Since position is coupled to momentum, as the object evolves in time, the backaction noise can perturb the position and contaminate subsequent position measurements. Scientists studying gravitational-wave detection, concerned that this dynamical effect of measurement backaction would place a fundamental limit to the detectors, proposed a general solution: If a quantum observable, represented by a self-adjoint operator $O(t)$ in the Heisenberg picture, can be made to commute with itself at times $t$ and $t^{\prime}$ when the observable is measured, viz.,

$$
\left[O(t), O\left(t^{\prime}\right)\right]=0
$$

then $O$ can be measured repeatedly with no quantum limits on the predictability of these measurements. In particular, this means that quantum mechanics does not limit the detection of a classical signal that affects $O$.

\footnotetext{
*eletmk@nus.edu.sg
}

Published by the American Physical Society under the terms of the Creative Commons Attribution 3.0 License. Further distribution of this work must maintain attribution to the author(s) and the published article's title, journal citation, and DOI.
An observable that obeys Eq. (1) is called a quantumnondemolition (QND) observable [1-4]. An observable that satisfies Eq. (1) at all times is called a continuoustime QND observable, and one that satisfies Eq. (1) only at discrete times is called a stroboscopic QND observable. The quantum backaction that accompanies measurements of $O$ directly affects observables that are conjugate to $O$; when Eq. (1) is satisfied, the conjugate observables do not feed back onto the QND observable at the times of interest.

The most well-known QND observables are ones that remain static in the absence of classical signals, viz.,

$$
O(t)=O\left(t^{\prime}\right)
$$

Peres showed that Eq. (2) is indeed a necessary condition for an observable to be QND in continuous time if $O(t)$ has a discrete spectrum (and has no explicit time dependence in the Schrödinger picture) [5]. Nowadays it is often assumed that Eqs. (1) and (2) are interchangeable as the QND condition [6-8]. Overemphasis on Eq. (2) as the QND condition trivializes the QND concept and has even led to calls for its retirement [8].

An assumption that Eq. (2) is a necessary QND condition implies that measurement backaction would always introduce additional uncertainties to any quantum system with richer dynamics than Eq. (2) and limit one's ability to process classical information accurately. A famous example of such thinking is the standard quantum limit to 
force sensing [1,2], which arises from backaction noise and was considered to be a fundamental limit on force sensitivity with position measurements.

The central result of this paper is to show that there exists a much wider class of observables that obey the QND condition, Eq. (1). To this end, we generalize the concept of a QND observable to that of a quantummechanics-free subsystem (QMFS) [9], which is a set of observables $\mathcal{O}=\left\{O_{1}, O_{2}, \ldots, O_{N}\right\}$ that obey, in the Heisenberg picture,

$$
\left[O_{j}(t), O_{k}\left(t^{\prime}\right)\right]=0 \quad \text { for all } j \text { and } k,
$$

at all times $t$ and $t^{\prime}$ (including $t=t^{\prime}$ ) when the observables are measured. Mathematically, Eq. (3) guarantees the classicality of a QMFS by virtue of the spectral theorem, which allows one to map the commuting Heisenbergpicture operators to processes in a classical probability space [10-12].

The correspondence between QND observables and classical processes implies that a QMFS is immune to the laws of quantum mechanics, such as the Heisenberg uncertainty principle and measurement invasiveness, at all times of interest. This exact classicality of a QMFS should be contrasted with the approximate classicality that emerges in the macroscopic limit through coarse graining or decoherence [13-15]. It should also be distinguished from classical simulability [16-19], which means that a quantum system has a complete description that can be efficiently implemented on a classical computer. A simulable quantum system can still suffer from measurement backaction.

Because any subset of a QMFS is also a QMFS, a decohering quantum system can contain a QMFS as well, if a set of system operators together with the environment operators form a larger QMFS. The environment then behaves as classical dissipation and fluctuation in the accessible part of the QMFS. In the same vein, we can broadly define measurements of any strength as QND if they can be dilated to projective measurements on a larger QMFS. Repeated QND measurements, even if they are projective on the system of interest, need not reproduce the same outcomes, as the QMFS can evolve during the measurements.

A continuous-time dynamical QMFS must consist of continuous variables, given Peres's result [5]. To construct such a system, consider two sets of canonical positions and momenta, $\{Q, P\}=\left\{Q_{1}, Q_{2}, \ldots, Q_{M}, P_{1}, P_{2}, \ldots, P_{M}\right\}$ and $\{\Phi, \Pi\}=\left\{\Phi_{1}, \Phi_{2}, \ldots, \Phi_{M}, \Pi_{1}, \Pi_{2}, \ldots, \Pi_{M}\right\}$, which obey the canonical commutation relations,

$$
\left[Q_{j}, P_{k}\right]=\left[\Phi_{j}, \Pi_{k}\right]=i \hbar \delta_{j k}
$$

and otherwise commute with one another. Suppose the Hamiltonian has the form

$$
H=\frac{1}{2} \sum_{j=1}^{M}\left(P_{j} f_{j}+f_{j} P_{j}+\Phi_{j} g_{j}+g_{j} \Phi_{j}\right)+h,
$$

where $f_{j}=f_{j}(Q, \Pi, t), \quad g_{j}=g_{j}(Q, \Pi, t), \quad$ and $\quad h=$ $h(Q, \Pi, t)$ are arbitrary, Hermitian-valued functions. The equations of motion for $Q_{j}(t)$ and $\Pi_{j}(t)$ in the Heisenberg picture become

$$
\dot{Q}_{j}=f_{j}(Q(t), \Pi(t), t), \quad \dot{\Pi}_{j}=-g_{j}(Q(t), \Pi(t), t) .
$$

The $Q$ and $\Pi$ variables are dynamically coupled to each other, but not to the incompatible set $\{\Phi, P\}$, and thus obey Eq. (3) and form a QMFS, as depicted in Fig. 1.

These QMFS variables can follow arbitrary classical trajectories in continuous time, including ones that are perturbed by classical signals or do not obey classical Hamiltonian dynamics. The QMFS variables can be prepared with arbitrarily small quantum uncertainties, or when monitored with sufficient accuracy, they will tend to such small quantum uncertainties. The measurement backaction acts on the conjugate variables $\{\Phi, P\}$. The resulting large quantum uncertainties required by the Heisenberg uncertainty principle are isolated in the variables $\{\Phi, P\}$, which do not influence the QMFS.

The classical trajectories followed within the QMFS do not have to obey Hamiltonian dynamics, but they will be those of a classical Hamiltonian $\tilde{H}(Q, \Pi, t)$ if we choose

$$
f_{j}=\frac{\partial \tilde{H}}{\partial \Pi_{j}}=\dot{Q}_{j}, \quad g_{j}=\frac{\partial \tilde{H}}{\partial Q_{j}}=-\dot{\Pi}_{j} .
$$

This QMFS was first suggested by Koopman as a formulation of classical Hamiltonian dynamics in a Hilbert space $[6,20]$, but its application to backaction evasion for quantum systems has not hitherto been appreciated.

A prime example of this sort of QMFS arises in the case of two pairs of canonical variables $(M=1)$ when the QMFS dynamics is that of a harmonic oscillator with

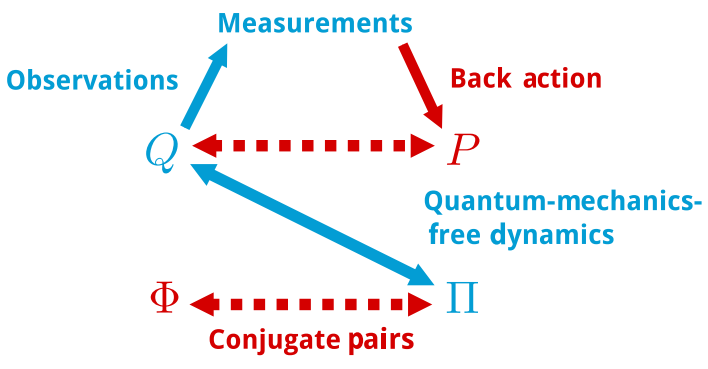

FIG. 1. A quantum-mechanics-free subsystem (QMFS, in blue), which consists of dynamically coupled quantumnondemolition (QND) observables $\{Q, \Pi\}$. The QMFS naturally evades measurement back-action; measurements of $Q$, for example, produce backaction onto the conjugate observable $P$, which does not influence the QMFS observables $Q$ and $\Pi$. 
mass $m$ and frequency $\omega$, i.e., classical Hamiltonian $\tilde{H}=$ $\Pi^{2} / 2 m+m \omega^{2} Q^{2} / 2$ and QMFS equations of motion:

$$
\dot{Q}=\frac{\Pi(t)}{m}, \quad \dot{\Pi}=-m \omega^{2} Q(t) .
$$

The overall quantum dynamics is that of the quadratic Hamiltonian

$$
H=\frac{P \Pi}{m}+m \omega^{2} \Phi Q,
$$

so $\{\Phi, P\}$ form an identical harmonic-oscillator QMFS in this case.

To get an idea of what this overall Hamiltonian means and how it might be implemented, we transform to new canonical variables,

$$
\begin{array}{ll}
Q=q+q^{\prime}, & P=\frac{p+p^{\prime}}{2}, \\
\Phi=\frac{q-q^{\prime}}{2}, & \Pi=p-p^{\prime},
\end{array}
$$

in terms of which the Hamiltonian (9) becomes

$$
H=\frac{p^{2}}{2 m}+\frac{1}{2} m \omega^{2} q^{2}-\frac{p^{\prime 2}}{2 m}-\frac{1}{2} m \omega^{2} q^{\prime 2}
$$

The transformed quantum system consists of two harmonic oscillators, one with positive mass and the other (primed) with negative mass. The relationships among the two sets of variables are summarized in Fig. 2.

A negative-mass oscillator is not the same as a particle moving in an inverted potential. Instead, the entire Hamiltonian is inverted. The dynamics consists of oscillations at frequency $\omega$, just as for a positive-mass oscillator,

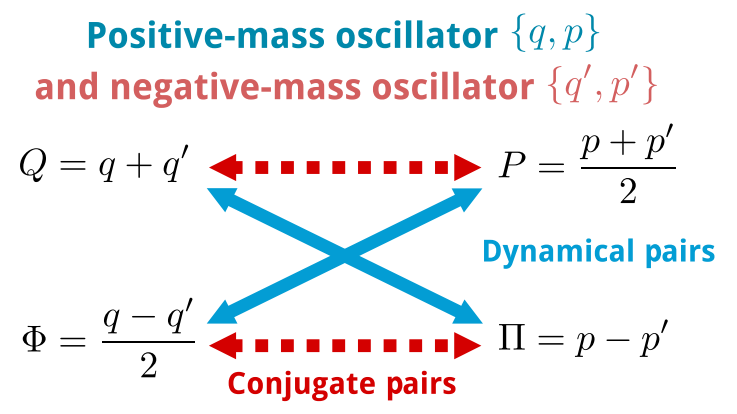

FIG. 2. Given two pairs of canonical variables, $\{q, p\}$ and $\left\{q^{\prime}, p^{\prime}\right\}$, the collective position $Q$ and average momentum $P$ form a conjugate pair, and the relative position $\Phi$ and relative momentum $\Pi$ form another conjugate pair. The conjugate pairs are restricted by the Heisenberg uncertainty principle. If the unprimed and primed variables are those of a positive-mass and negative-mass oscillator, respectively, however, $\{Q, \Pi\}$ and $\{\Phi, P\}$ form dynamical oscillator pairs, each of which is a QMFS. Reducing uncertainty in one QMFS at the expense of the other is equivalent to introducing Einstein-Podolsky-Rosen (EPR) correlations among the original oscillator variables. and the energy eigenstates are the same as those of a positive-mass oscillator, but the ladder of energy levels runs down instead of up; each quantum of excitation reduces the oscillator energy by $\hbar \omega$. For a negative-mass oscillator, creating a quantum of excitation involves extracting energy $\hbar \omega$, instead of supplying that amount of energy. Despite not being just an inverted potential, negative-mass oscillators are sometimes referred to as inverted oscillators [21,22].

The continuous-time QMFS of Eqs. (8)-(12) is naturally backaction evading [1-3], as measurements of $Q(t)$ or $\Pi(t)$ introduce backaction to the conjugate variables $P(t)$ or $\Phi(t)$, which are never coupled to the measured subsystem. A complementary perspective is to consider the QMFS as a quantum noise-cancellation scheme [23]: Measurements of $Q=q+q^{\prime}$ produce equal backaction onto $p$ and $p^{\prime}$, which cancels coherently in the dynamical variable, $\Pi=$ $p-p^{\prime}$, that is coupled to $Q$. Quantum noise cancellation is illustrated in Fig. 3.

A pairing of positive- and negative-mass oscillators occurs naturally as mirror sidebands of a carrier frequency $\Omega$. Thus consider two field modes, with frequencies $\Omega \pm \omega$ placed symmetrically about $\Omega$. The Schrödinger-picture Hamiltonian of the two modes is $H_{\mathrm{SP}}=\hbar(\Omega+\omega) a^{\dagger} a+\hbar(\Omega-\omega) b^{\dagger} b$, where $a$ and $b$ are annihilation operators for the blue and red sidebands. If energies are defined relative to the carrier frequency, creating a quantum of excitation in the blue sideband requires energy $\hbar \omega$, whereas creating a quantum of excitation in the red sideband yields $\hbar \omega$. To see this formally, we transform to the modulation picture [24], which moves the rapid oscillation at the carrier frequency from quantum states to operators. If we explicitly remove this rapid oscillation from the annihilation operators,

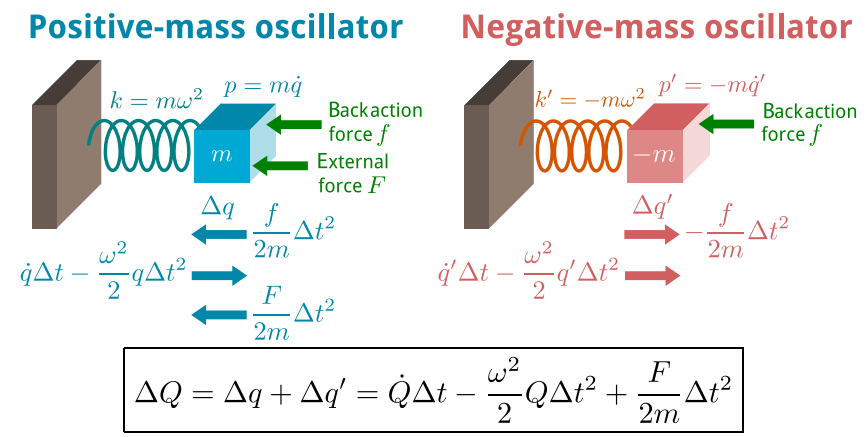

FIG. 3. Behavior of positive-mass (blue) and negative-mass (red) oscillators during a short time interval $\Delta t$. Monitoring the collective position $Q$ leads to the same backaction force $f$ on both oscillators. The positive-mass oscillator is "pushed" by $f$, whereas the negative-mass oscillator is "pulled" in the opposite direction; the effect of the backaction thus cancels in $Q$. The evolution of $Q$ under an external force $F$ applied to the positive-mass oscillator is exactly the same as that of a single oscillator. Positive- and negative-mass oscillators can be realized as blue and red sidebands of a carrier frequency. 


$$
a e^{i \Omega t}=\sqrt{\frac{\omega}{2 \hbar}}\left(q+\frac{i p}{\omega}\right), \quad b e^{i \Omega t}=\sqrt{\frac{\omega}{2 \hbar}}\left(q^{\prime}+\frac{i p^{\prime}}{\omega}\right)
$$

we end with a pair of oscillators that oscillate at the modulation frequency $\omega$. The blue sideband is a positive-mass oscillator, and the red sideband is a negative-mass oscillator. The modulation-picture Hamiltonian, $H_{\mathrm{MP}}=\hbar \omega\left(a^{\dagger} a-b^{\dagger} b\right)$, is the twooscillator Hamiltonian (12) with $m=1$.

To illustrate the connection to the QMFS variables, it is instructive to introduce an electromagnetic field operator given by

$$
E=E^{(+)}+E^{(-)}=E_{1} \cos \Omega t+E_{2} \sin \Omega t .
$$

Here,

$$
E^{(+)}=\sqrt{\frac{\hbar \Omega}{2}}(a+b)=\frac{1}{2}\left(E_{1}+i E_{2}\right) e^{-i \Omega t}
$$

and $E^{(-)}=E^{(+) \dagger}$ are the positive- and negative-frequency parts of the field, and $E_{1}$ and $E_{2}$ are the field's (Hermitian) quadrature components, defined relative to the carrier frequency. The quadrature components take the form $E_{1}=\sqrt{\hbar \Omega}\left(\alpha_{1}+\alpha_{1}^{\dagger}\right)$ and $E_{2}=\sqrt{\hbar \Omega}\left(\alpha_{2}+\alpha_{2}^{\dagger}\right)$, where

$$
\begin{gathered}
\alpha_{1}=\frac{1}{\sqrt{2}}\left(a e^{i \Omega t}+b^{\dagger} e^{-i \Omega t}\right)=\frac{1}{2} \sqrt{\frac{\omega}{\hbar}}\left(Q+i \frac{\Pi}{\omega}\right), \\
\alpha_{2}=-\frac{i}{\sqrt{2}}\left(a e^{i \Omega t}-b^{\dagger} e^{-i \Omega t}\right)=\sqrt{\frac{\omega}{\hbar}}\left(-i \Phi+\frac{P}{\omega}\right)
\end{gathered}
$$

are the quadrature amplitudes [24-26]. Just as the annihilation operators $a$ and $b$ are quantum operators for the classical variables that encode the amplitude and phase of the modal oscillations at frequencies $\Omega \pm \omega$, so the quadrature amplitudes $\alpha_{1}$ and $\alpha_{2}$ encode the amplitude and phase of the oscillations of the quadrature components. Each quadrature amplitude describes oscillations within a QMFS.

Two-mode squeezed states $[24,26]$ take advantage of this QMFS structure in an electromagnetic wave to decrease the quantum uncertainties associated with one quadrature component, while increasing the uncertainty associated with the other. Homodyne detection at the carrier frequency [25] measures one quadrature component and, hence, the signal within a QMFS. The broadband squeezed states now being introduced into interferometric gravitational-wave detectors [27,28] use two-mode squeezing over a wide bandwidth of modulation frequencies and are thus an example of using dynamical QMFSs in probing the motion of a mechanical system. In such broadband squeezed states, the $\{Q, \Pi\}$ variables are not correlated with the $\{\Phi, P\}$ set, but the oscillator variables for the blue and red sidebands are necessarily correlated in the Einstein-Podolsky-Rosen (EPR) sense [17].

These considerations suggest a way to implement a QMFS using a mechanical oscillator. If the oscillator is probed by an optical beam with carrier frequency $\Omega$, the negative-mass harmonic oscillator can be simulated by an optical mode in a cavity with a red-detuned resonance at $\Omega-\omega$ [23]. This strategy of introducing an auxiliary mode to form a QMFS and measuring the collective position $Q$ enables one to beat the standard quantum limit for force detection [23,29]. It also allows one to entangle the mechanical oscillator with the auxiliary mode, as has been proposed in a scenario where the role of the red-detuned auxiliary mode is played by a polarized atomic spin ensemble [30].

Another way of implementing Eq. (12) is to use two spin ensembles, both of which have total angular momentum $J_{0}$. Suppose the ensembles are polarized nearly maximally, but oppositely along the direction of an applied magnetic field $B_{0} \mathbf{e}_{z}$. The average angular momenta are then $\langle\boldsymbol{J}\rangle=$ $-\left\langle\boldsymbol{J}^{\prime}\right\rangle \simeq J_{0} \mathbf{e}_{z}$. Off-axis polarizations precess about the magnetic field. For large angular momentum, the precessional oscillations of the $x$ and $y$ components of the angular momenta are identical to the phase-space trajectory of a harmonic oscillator. Moreover, the aligned angular momentum $\boldsymbol{J}$ has magnetic sublevels whose energy increases away from maximal polarization, making it a positivemass oscillator, whereas for the antialigned angular momentum $\boldsymbol{J}^{\prime}$, the magnetic sublevels decrease in energy, making it a negative-mass oscillator. The resulting QMFS structure has been used to achieve quantum noise cancellation [31,32].

Formally, we have, in the Holstein-Primakoff approximation,

$$
\begin{gathered}
{\left[J_{x}, J_{y}\right]=i \hbar J_{z} \simeq i \hbar J_{0},} \\
{\left[J_{x}^{\prime}, J_{y}^{\prime}\right]=i \hbar J_{z}^{\prime} \simeq-i \hbar J_{0} .}
\end{gathered}
$$

Defining canonical position and momentum operators by

$$
\begin{aligned}
q=J_{x} / \sqrt{J_{0}}, & p=J_{y} / \sqrt{J_{0},} \\
q^{\prime}=J_{x}^{\prime} / \sqrt{J_{0}}, & p^{\prime}=-J_{y}^{\prime} / \sqrt{J_{0},}
\end{aligned}
$$

and using $J_{z} \simeq \sqrt{J_{0}\left(J_{0}+1\right)}-\left(q^{2}+p^{2}\right) / 2$ and $J_{z}^{\prime} \simeq$ $-\sqrt{J_{0}\left(J_{0}+1\right)}+\left(q^{\prime 2}+p^{\prime 2}\right) / 2$, the Hamiltonian becomes

$$
H=-\gamma B_{0}\left(J_{z}+J_{z}^{\prime}\right) \simeq \frac{\gamma B_{0}}{2}\left(q^{2}+p^{2}-q^{\prime 2}-p^{\prime 2}\right),
$$

which has the form of Eq. (12).

Since $Q$ and $\Pi$ commute at all times, continuous measurements of one reveal information about the other with no backaction, and the pair can have uncertainties whose product is less than the minimum that the Heisenberg 
uncertainty principle allows for conjugate variables. As noted above, this sub-Heisenberg uncertainty product means that the two physical oscillators, $\{q, p\}$ and $\left\{q^{\prime}, p^{\prime}\right\}$, are entangled in the EPR sense. The collectiveangular-momentum experimental demonstration of entanglement in [31] can thus be regarded as a demonstration of a QMFS that behaves as a classical harmonic oscillator. Moreover, the magnetometer reported in [32] demonstrates the use of a dynamical QMFS for sensing that does not suffer from quantum-measurement backaction. The dynamical QMFS ( $\{\Phi, P\}$ in this case) has the advantage of being resonant with oscillating magnetic-field signals in the $x$ or $y$ direction near the tunable Larmor frequency $\gamma B_{0}$, whereas a static QMFS with operators that obey Eq. (2) is much less sensitive to oscillating signals when the signal phase is unknown.

It is possible to construct discrete-variable QMFSs as well, as long as the QND condition is imposed stroboscopically. Examples come from quantum computation. Suppose we have a collection of $N$ qubits with Pauli $Z$ operators given by $\left\{Z_{1}, \ldots, Z_{N}\right\}$ [33]. The simultaneous eigenstates of the Pauli $Z$ operators for all the qubits are specified by bit strings $a_{1}, \ldots, a_{N}$, where $(-1)^{a_{j}}$ is the eigenvalue of $Z_{j}$ for qubit $j$. These eigenstates $\left|a_{1}, \ldots, a_{N}\right\rangle$ are called the computational-basis states. A quantum gate that permutes computational-basis states executes a classical (reversible) gate on the input bit string. In the Heisenberg picture, such a gate takes the input $Z$ operators to output $Z$ operators that are functions of the input $Z$ operators and thus commute with them. The classical information processing performed by the gate can be regarded as noiseless information processing performed within the QMFS of the Pauli $Z$ operators restricted to times pre- and postgate.

An example of such a gate is the controlled-NOT gate [16], which transforms the computational-basis states according to $|a, b\rangle \rightarrow\left|a^{\prime}, b^{\prime}\right\rangle=|a, b \oplus a\rangle$, where $\oplus$ denotes binary addition and primes denote postgate values. The corresponding Heisenberg-picture transformation of the Pauli $Z$ operators is $Z_{1}^{\prime}=Z_{1}$ and $Z_{2}^{\prime}=Z_{1} Z_{2}$. A more ambitious example is the three-qubit Toffoli gate [16,34,35], a controlled-controlled-NOT gate, which transforms computational-basis states as $|a, b, c\rangle \rightarrow$ $\left|a^{\prime}, b^{\prime}, c^{\prime}\right\rangle=|a, b, c \oplus a b\rangle$ in the Schrödinger picture and transforms the Pauli $Z$ operators in the Heisenberg picture according to

$$
\begin{aligned}
& Z_{1}^{\prime}=Z_{1}, \quad Z_{2}^{\prime}=Z_{2}, \\
& Z_{3}^{\prime}=\left[I-\frac{1}{2}\left(I-Z_{1}\right)\left(I-Z_{2}\right)\right] Z_{3},
\end{aligned}
$$

where $I$ is the identity operator. For both these gates, since the output $Z$ operators commute with the input, the $Z$ operators can be mapped to the classical bits of the computational basis, which undergo classical information processing between input and output and can be measured at any stage without spoiling the computation [36].

Classical Toffoli gates form a set of universal gates for (reversible) classical computation [34], so one can construct any classical discrete-variable dynamics in discrete time using a circuit of quantum Toffoli gates. Thus, Benioff and Feynman's quantum-mechanical computer for universal classical computation $[35,37,38]$ is an example of information processing within a dynamical QMFS. Experimental demonstrations of the quantum Toffoli gate have been reported in [39-41].

The existence of QMFSs does not contradict proven quantum limits to classical information processing, such as the quantum Cramér-Rao bound on waveform estimation $[29,42,43]$ and the Helstrom bound on waveform detection [42-44], as all such limits are derived from quantum mechanics. This implies that proven quantum limits should either involve incompatible observables outside a QMFS or have effectively classical origins.

In practice, of course, it is generally difficult even to get to backaction-enforced standard quantum limits, and, if one is trying to go well beyond such limits by using the observables of a QMFS, imperfections, losses, and decoherence will make it quite difficult to avoid contamination of the QMFS observables with the necessarily large quantum noise in the incompatible observables. Such practical limitations do not, however, affect our conclusion that QMFSs can have exactly classical dynamics and be entirely free of quantum backaction.

The concept of a QMFS unifies under a single framework the several strategies for evading measurement backaction, such as QND observables, backaction evasion, and quantum noise cancellation. Given what we have seen from the example of force sensing, where a QMFS can beat the standard quantum limit and approach the quantum CramérRao and Helstrom bounds [23,29,44], we envision QMFSs to be a useful tool for overcoming heuristic quantum limits and approaching proven limits for classical informationprocessing applications in general.

We acknowledge useful discussions with Joshua Combes. This material is based on work supported in part by the Singapore National Research Foundation under NRF Grant No. NRF-NRFF2011-07, U.S. National Science Foundation Grant Nos. PHY-0903953 and PHY1005540, and U.S. Office of Naval Research Grant No. N00014-11-1-0082.

[1] V. B. Braginsky and F. Ya. Khalili, Quantum Measurement (Cambridge University Press, Cambridge, England, 1992).

[2] C. M. Caves, K. S. Thorne, R.W.P. Drever, V.D. Sandberg, and M. Zimmermann, On the Measurement of a Weak Classical Force Coupled to a QuantumMechanical Oscillator. I. Issues of Principle, Rev. Mod. Phys. 52, 341 (1980). 
[3] V. B. Braginsky, Y.I. Vorontsov, and K.S. Thorne, Quantum Nondemolition Measurements, Science 209, 547 (1980).

[4] W. G. Unruh, Quantum Nondemolition and Gravity-Wave Detection, Phys. Rev. D 19, 2888 (1979).

[5] A. Peres, Quantum Limited Detectors for Weak Classical Signals, Phys. Rev. D 39, 2943 (1989).

[6] A. Peres, Quantum Theory: Concepts and Methods (Kluwer Academic, Dordrecht, Netherlands, 2002).

[7] H. M. Wiseman and G. J. Milburn, Quantum Measurement and Control (Cambridge University Press, Cambridge, England, 2010).

[8] C. Monroe, Demolishing Quantum Nondemolition, Phys. Today 64, 8 (2011).

[9] The name is inspired by the use of decoherence-free subspaces or subsystems to describe subspaces or subsystems that do not see the effects of particular types of decoherence; see D. A. Lidar and K. B. Whaley, in Irreversible Quantum Dynamics, edited by F. Benatti and R. Floreanini, Lecture Notes in Physics Vol. 622 (Springer, Berlin, 2003), pp. 83-120. Although the name is similar, the concept here is different. We use QMFS to emphasize the exact classical dynamics of the observables in a QMFS, which is to be contrasted with the approximate classical dynamics that emerges as a consequence of decoherence or in a classical limit.

[10] M. Reed and B. Simon, Methods of Modern Mathematical Physics. I: Functional Analysis (Academic Press, San Diego, 1980).

[11] V.P. Belavkin, Nondemolition Principle of Quantum Measurement Theory, Found. Phys. 24, 685 (1994).

[12] L. Bouten, R. van Handel, and M. R. James, An Introduction to Quantum Filtering, SIAM J. Control Optim. 46, 2199 (2007).

[13] W. H. Zurek, Decoherence, Einselection, and the Quantum Origins of the Classical, Rev. Mod. Phys. 75, 715 (2003).

[14] M. Schlosshauer, Decoherence and the Quantum-toClassical Transition (Springer, Berlin, 2007).

[15] G. J. Milburn, Decoherence and the Conditions for the Classical Control of Quantum Systems, arXiv:1201.5111.

[16] M. A. Nielsen and I.L. Chuang, Quantum Computation and Quantum Information (Cambridge University Press, Cambridge, England, 2000).

[17] S. L. Braunstein and P. van Loock, Quantum Information with Continuous Variables, Rev. Mod. Phys. 77, 513 (2005).

[18] S.D. Bartlett, T. Rudolph, and R.W. Spekkens, Reconstruction of Gaussian Quantum Mechanics from Liouville Mechanics with an Epistemic Restriction, Phys. Rev. A 86, 012103 (2012).

[19] Examples of classically simulable systems include continuous-variable systems with initial nonnegative Wigner representations, a quadratic Hamiltonian with respect to canonical positions and momenta, and measurements of phase-space observables $[7,17,18]$ and collections of qubits with state preparation in the computational basis, Clifford-group gates, and measurements of Pauligroup observables (Gottesman-Knill theorem [16]).

[20] B.O. Koopman, Hamiltonian Systems and Transformations in Hilbert Space, Proc. Natl. Acad. Sci. U.S.A. 17, 315 (1931).
[21] R. J. Glauber, in New Techniques and Ideas in Quantum Measurement Theory, Ann. New York Acad. Sci. Vol. 480, edited by D. M. Greenberger (New York Academy of. Science, New York, 1986), pp. 336-372.

[22] C. W. Gardiner and P. Zoller, Quantum Noise: A Handbook of Markovian and Non-Markovian Quantum Stochastic Methods with Applications to Quantum Optics (Springer, New York, 2004), 3rd ed.

[23] M. Tsang and C. M. Caves, Coherent Quantum-Noise Cancellation for Optomechanical Sensors, Phys. Rev. Lett. 105, 123601 (2010).

[24] C. M. Caves and B.L. Schumaker, New Formalism for Two-Photon Quantum Optics. I. Quadrature Phases and Squeezed States, Phys. Rev. A 31, 3068 (1985). In observable field operators, such as the electric field of an electromagnetic wave, the $\sqrt{\Omega}$ dependence in Eq. (15) is replaced by a square root of the mode frequency. This makes a negligible difference when the carrier frequency is much higher than the modulation frequency; see also [25].

[25] J.H. Shapiro and S.S. Wagner, Phase and Amplitude Uncertainties in Heterodyne Detection, IEEE J. Quantum Electron. 20, 803 (1984).

[26] B.L. Schumaker and C. M. Caves, New Formalism for Two-Photon Quantum Optics. II. Mathematical Foundation and Compact Notation, Phys. Rev. A 31, 3093 (1985).

[27] M. Mehmet, H. Vahlbruch, N. Lastzka, K. Danzmann, and R. Schnabel, Observation of Squeezed States with Strong Photon-Number Oscillations, Phys. Rev. A 81, 013814 (2010).

[28] J. Abadie et al. (LIGO Scientific Collaboration), A Gravitational-Wave Observatory Operating beyond the Quantum Shot-Noise Limit, Nature Phys. 7, 962 (2011).

[29] M. Tsang, H. M. Wiseman, and C. M. Caves, Fundamental Quantum Limit to Waveform Estimation, Phys. Rev. Lett. 106, 090401 (2011).

[30] K. Hammerer, M. Aspelmeyer, E. S. Polzik, and P. Zoller, Establishing Einstein-Podolsky-Rosen Channels between Nanomechanics and Atomic Ensembles, Phys. Rev. Lett. 102, 020501 (2009).

[31] B. Julsgaard, A. Kozhekin, and E. S. Polzik, Experimental Long-Lived Entanglement of Two Macroscopic Objects, Nature (London) 413, 400 (2001).

[32] W. Wasilewski, K. Jensen, H. Krauter, J. J. Renema, M. V. Balabas, and E.S. Polzik, Quantum Noise Limited and Entanglement-Assisted Magnetometry, Phys. Rev. Lett. 104, 133601 (2010).

[33] Each of these qubits can be a "logical" qubit and consist of multiple physical qubits [16]. Much like the continuous-variable case, where the QMFS observables can be collective degrees of freedom and measurements can create EPR entanglement, $Z_{j}$ can be a product of Pauli operators for multiple physical qubits, and measurements of $Z_{j}$ can lead to entanglement of the physical qubits. For example, two physical qubits with Pauli operators $\left\{\sigma_{x 1}, \sigma_{z 1}, \sigma_{x 2}, \sigma_{z 2}\right\}$ can be entangled to form a Bell state by measuring the commuting operators $Z_{1} \equiv \sigma_{x 1} \sigma_{x 2}$ and $Z_{2} \equiv \sigma_{z 1} \sigma_{z 2}$.

[34] T. Toffoli, in Proceedings of the 7th Colloquium on Automata, Languages and Programming, edited by J. 
de Bakker and J. van Leeuwen Lecture Notes in Computer Science Vol. 85 (Springer, Berlin, 1980), pp. 632-644.

[35] R.P. Feynman, Quantum Mechanical Computers, Opt. News 11, 11 (1985).

[36] See M. Ozawa, Quantum Nondemolition Monitoring of Universal Quantum Computers, Phys. Rev. Lett. 80, 631 (1998) for an example of how the concept of QND observables can also be useful for the monitoring of quantum computers.

[37] P. Benioff, The Computer as a Physical System: A Microscopic Quantum-Mechnical Hamiltonian Model of Computers as Represented by Turing Machines, J. Stat. Phys. 22, 563 (1980).

[38] P. Benioff, Quantum-Mechanical Hamiltonian Models of Turing Machines, J. Stat. Phys. 29, 515 (1982).

[39] T. Monz, K. Kim, W. Hänsel, M. Riebe, A. S. Villar, P. Schindler, M. Chwalla, M. Hennrich, and R. Blatt,
Realization of the Quantum Toffoli Gate with Trapped Ions, Phys. Rev. Lett. 102, 040501 (2009).

[40] A. Fedorov, L. Steffen, M. Baur, M. P. da Silva, and A. Wallraff, Implementation of a Toffoli Gate with Superconducting Circuits, Nature (London) 481, 170 (2011).

[41] M. D. Reed, L. DiCarlo, S. E. Nigg, L. Sun, L. Frunzio, S. M. Girvin, and R. J. Schoelkopf, Realization of ThreeQubit Quantum Error Correction with Superconducting Qubits, Nature (London) 482, 382 (2012).

[42] C.W. Helstrom, Quantum Detection and Estimation Theory (Academic Press, New York, 1976).

[43] A.S. Holevo, Probabilistic and Statistical Aspects of Quantum Theory (North-Holland, Amsterdam, 1982).

[44] M. Tsang and R. Nair, Fundamental Quantum Limit to Waveform Detection, arXiv:1204.3697. 